\title{
A replication of the face-superiority effect
}

\author{
JAN P. H. van SANTEN and JOHN JONIDES \\ University of Michigan, Ann Arbor, Michigan 48109
}

\begin{abstract}
Schematic faces, scrambled faces (stimuli differing from schematic faces in that the positions of the eyes, noses, and mouths were anomalous, but constant from trial to trial), and individual face components were presented in a tachistoscope followed by a patterned mask. Following the mask, either four alternative mouths or four alternative pairs of eyes were presented, and subject3 were required to choose among the alternatives for the feature that had been presented in the tachistoscopic display. Performance was best when the original display contained only individual component features, and it was worst when scrambled faces were stimuli, thus replicating a similar experiment by Homa, Haver, and Schwartz (1976). Contrary to their findings, however, the effect of feature scrambling was greater on recognition of eyes than on mouths.
\end{abstract}

Homa, Haver, and Schwartz (1976) have recently found better recognition performance on schematic faces than on scrambled versions of these schematic faces in which the head outline contained, from top to bottom, a nose, a mouth, and a pair of eyes. In addition, they found that performance was best when the face components (nose, mouth, eyes) were presented in isolation. These effects were demonstrated using a forced-choice recognition procedure in which the impact of sophisticated guessing was removed by equally combining all alternative feature instances. It would seem that the effect due to scrambling demonstrated in this work belongs to a class of similar effects that have been under study recently, including word-superiority effects (e.g., Reicher, 1969), effects of scrambling scenes (Biederman, Glass, \& Stacy, 1973), and objectsuperiority effects (e.g., Weisstein \& Harris, 1974). While these other phenomena have received considerable empirical attention, however, the effect of scrambling on the recognition of facial features has not. In the present study, therefore, we sought to better establish the empirical base of this effect by replicating and extending the basic experiment of Homa et al. (1976).

\section{METHOD}

\section{Subjects}

The subjects were 36 undergraduate students from the University of Michigan. Participation in the experiment was part of a course requirement.

\section{Stimuli}

Sixteen schematic faces and sixteen scrambled versions of these faces were constructed from a set of four mouths, four pairs of eyes, and one nose (roughly looking like an inversion of

This research was conducted while the first author was supported by a Horace H. Rackham predoctoral fellowship from the University of Michigan. The research was also partially supported by National Science Foundation Grant BNS77-16887 to the second author. We thank Donald Homa for permission to use his stimuli in a portion of this study. the digit 7 , subtending a visual angle of about $.41 \mathrm{deg})$. The pairs of eyes and the mouths were reproductions (black-and-white line drawings) of parts of highly emotional faces used in a number of cross-cultural studies on emotion identification reported by Ekman (1973). The head outlines had no ears, were ellipsoid in shape, and had height and width of 2.67 and $2.11 \mathrm{deg}$, respectively. In the face stimuli, the distance between the center of the eyes and the center of the mouth was $1.08 \mathrm{deg}$. The corresponding distance in the scrambled-face stimuli was $.79 \mathrm{deg}$.

\section{Design}

Two types of trial blocks were constructed: blocks of faces and scrambled faces, and blocks in which isolated components were presented individually. On each trial in both types of blocks, the subject had to choose among either four alternative mouths or four alternative pairs of eyes after viewing the stimulus. Of course, these alternatives were the same components as were used for stimulus construction. Each block of the face/scrambled-face variety consisted of 64 trials, 32 with each type of stimulus using all combinations of eye and mouth features (once testing for the correct alternative eyes, once for the mouth). Each block of isolated components consisted of 16 trials, two presentations each of the eight possible component eyes or mouths. Each subject was given four face/scrambled-face blocks (the first was a practice block) and three individual component blocks such that the blocks of one type were alternated with those of the other type. The total number of trials in the experiment was 304 for each subject.

\section{Procedure}

The stimuli were presented in a three-field tachistoscope (Scientific Prototype, Model GB). Each face/scrambled-face trial proceeded as follows: A schematic head outline served as a fixation frame; it had the same location and size as the outline of the stimuli, and it was visible between trials. After the experimenter gave an auditory signal, the subject pressed a switch, which, after $500 \mathrm{msec}$, caused the offset of the fixation frame and the simultaneous onset of the stimulus. Immediately after the offset of the stimulus, a mask was presented for 2,000 msec. The mask consisted of a network of lines and curves having the same width as the lines used for drawing the stimuli. It extended about $3 \mathrm{deg}$ both horizontally and vertically. Simultaneous with the offset of the mask, either four mouths or four pairs of eyes were shown with one of the numbers 1 through 4 printed above each. The subject's task was to respond verbally with the number corresponding to what he considered the correct alternative. After the response, the card containing the alternatives was removed so that the fixation frame became visible again. On the 
individual component trials, the procedure was identical. The luminance in each of the three fields was $77 \mathrm{ftL}$.

Each session lasted 1-1.5 h and began with a practice face/ scrambled-face block. During this block, the exposure duration was reduced from $150 \mathrm{msec}$ to a duration at which the subject was correct on $60 \%-70 \%$ of the trials. This final exposure duration ranged between 60 and $110 \mathrm{msec}$ across subjects, with an average of $85 \mathrm{msec}$. The exposure duration of the first individual component block was set at about $60 \%$ of the final exposure duration of the practice face/scrambled-face block. Between blocks, the exposure duration was adjusted so as to keep the performance between $60 \%$ and $70 \%$ correct. The average duration over the three test face/scrambled-face blocks ranged between 56.7 and $113.3 \mathrm{msec}$, with an average of $80.9 \mathrm{msec}$. The corresponding range for the individual component blocks was 26.7 to $61.7 \mathrm{msec}$, with an average of 38.5.

\section{RESULTS}

Overall percentages correct for eyes in faces, eyes in scrambled faces, mouths in faces, and mouths in scrambled faces were 73.8, 52.9, 78.0, and 71.1, respectively. Eyes and mouths, when presented individually, were identified correctly on $65.9 \%$ and $77.0 \%$ of the trials, respectively. The data from the face/scrambled-face blocks were analyzed separately from the data obtained in the individual component blocks.

The face/scrambled-face data form a complete 16 by 36 by 2 by 2 design, with two random factors (eye-mouth combinations and subjects) and two fixed factors (face vs. scrambled face, and testing of eyes vs. mouth). Following Clark (1973, 1976), an analysis of variance in this design is equivalent to using $t$ tests for effects of which the standard error depends on two separately estimated standard errors. Tables for this t statistic are provided by Aspin (1947), and by Trickett, Welch, and James (1956). The statistic is calculated by dividing the effect by the square root of $\mathrm{SE}_{\mathrm{s}}^{2}+\mathrm{SE}_{\mathrm{c}}^{2}$, where $\mathrm{SE}_{\mathrm{s}}$ is the standard error of the effect calculated over subjects and $\mathrm{SE}_{\mathrm{c}}$ is the standard error of the effect calculated over eye-mouth combinations. We will use $t(35,15)$ to denote this statistic with 35 (for subjects) and 15 (for eye-mouth combinations) degrees of freedom. The letter "c" will be used for $\mathrm{SE}_{\mathrm{s}}^{2} /\left(\mathrm{SE}_{\mathrm{s}}^{2}+\mathrm{SE}_{\mathrm{c}}^{2}\right)$. This parameter reflects the ratio of the two standard errors and determines, together with the degrees of freedom, which entry of the tables should be consulted. We will use $t_{s}(35)$ for the ordinary $t$ test treating subjects as the only random factor and $t_{c}(15)$ for such tests treating eye-mouth combinations as the only random factor.

According to this analysis scheme, the results were as follows. The effect of scrambling: $t(35,15)=6.16$, $\mathrm{c}=.542, \mathrm{p}<.005 ; \mathrm{t}_{\mathrm{s}}(35)=8.36, \mathrm{p}<.001 ;$ and $\mathrm{t}_{\mathrm{c}}(15)$ $=9.10, \mathrm{p}<.001$ (all one-tailed tests). The interaction of scrambling with component tested: $\mathrm{t}(35,15)=2.43$, $\mathrm{c}=.509, \mathrm{p}<.04 ; \mathrm{t}_{\mathrm{s}}(35)=3.29, \mathrm{p}<.005 ;$ and $\mathrm{t}_{\mathrm{c}}(15)$ $=3.35, \mathrm{p}<.005$ (all two-tailed tests). In addition, the effect of scrambling was tested for the eyes and the mouths separately. For the eyes: $\mathrm{t}(35,15)=5.07$, $\mathrm{c}=.373, \mathrm{p}<.005 ; \mathrm{t}_{\mathrm{s}}(35)=8.29, \mathrm{p}<.001 ;$ and $\mathrm{t}_{\mathrm{c}}(15)$ $=6.39, \mathrm{p}<.001$ (all one-tailed tests). For the mouths: $\mathrm{t}(35,15)=2.08, \mathrm{c}=.751, \mathrm{p}<.025 ; \mathrm{t}_{\mathrm{s}}(35)=2.40$, $\mathrm{p}<.015 ; \mathrm{t}_{\mathrm{c}}(15)=4.16, \mathrm{p}<.001$ (all one-tailed tests).

The $11.1 \%$ difference in performance between mouths and eyes presented in isolation was reliable over subjects $\left[\mathrm{t}_{\mathrm{s}}(35)=4.77, \mathrm{p}<.001\right.$, two-tailed test] However, one of the mouths was perceived less accurately than three of the four pairs of eyes.

Although the experiment was not designed to compare performance on the two types of stimulus blocks, there is no doubt that the individual component trials were much easier than the face trials. One indication of this is the fact that the difference in mean exposure duration between the two types of blocks was $42.5 \mathrm{msec}$ (the smallest difference for any subject being $13 \mathrm{msec}$ ). To evaluate this difference in exposure duration, note that the difference in performance on mouths in faces compared to mouths presented in isolation, for example, was negligible $\left[\mathrm{t}_{\mathrm{s}}(35)=.35, \mathrm{p}>.25\right.$, one-tailed test $]$.

\section{DISCUSSION}

The present findings replicate the main results of Homa et al. (1976), in that performance was better on faces than on scrambled faces and best when the components were presented individually. There is one discrepancy between the present results and those of Homa et al. (1976), however. This is the stronger effect of scrambling on eyes than on mouths. In more than half of the experimental conditions used by Homa et al. (1976), the opposite was found. It would seem unlikely that this discrepancy is due to the fact that different pairs of eyes, mouths, and noses were used, because a pilot study by the first author, using four of the five mouths and pairs of eyes employed by Homa et al. (1976), found the same interaction as the present experiment (the pair of eyes and the mouth not used in the pilot study were perceived with the same accuracy as the other mouths and pairs of eyes).

There could be at least two causes for this discrepancy. First, it might have to do with the fact that the overall performance levels in the present study and in the pilot study were higher by about $20 \%$ than the performance level in the study by Homa et al. (1976). It simply could be that the perceptibility of eyes in faces increases faster when exposure duration increases than the perceptibility of eyes in scrambled faces, but that no such effect occurs for mouths.

A second possibility is that, for some unspecified reason, subjects in our experiment tended to focus on the upper half of the display. This would give eyes in faces an advantage over eyes in scrambled faces, but mouths in faces would not have as great an advantage over mouths in scrambled faces, since their relative locations were more similar than were those for the eyes.

Whatever the reason for the discrepancy between our results and those of Homa et al. (1976), our experiment does replicate their major finding of a superiority in recognizing features when they occur in faces as compared to scrambled faces.

There appear to be at least three explanations of this effect of scrambling. First, it could be due entirely to visual artifacts. For example, in the experiment of Homa et al. (1976), the location of the nose was physically more peripheral in the scrambled faces than in the schematic faces. A second possibility is that the configuration of the components could interact with the formation of a visual representation of the stimulus. That is, 
the configuration could affect the quality of the visual representation of the components that must be accessed for the subject to reach a decision. A special case of this could arise, for example, if there were location-specific detectors for mouth, eyes, and nose, so that an eye detector, say, would malfunction when confronted with a nose. A third possibility is that the visual representation for faces compared to that for scrambled faces is recoded into a more abstract representation that is less susceptible to decay and interference caused by the mask or by response processes. This recoding process would, by hypothesis, be more efficient for faces than for scrambled faces. This last explanation is suggested by several findings involving effects of face inversion that seem to indicate that normal adults have a specific capacity for encoding noninverted as opposed to inverted faces (e.g., Yin, 1969; Carey-Block, Note 1). It is clear that further research is needed to disentangle these alternatives.

\section{REFERENCE NOTE}

1. Carey-Block, S. A case study: Face recognition. In Explorations in the biology of language. Report of the MIT work group in the biology of language. Unpublished manuscript, MIT, 1976.

\section{REFERENCES}

Aspin, A. A. Tables for use in comparisons whose accuracy involves two variances, separately estimated. Biometrika, 1947, 36, 290-293.
Biederman, I.. Glass, A. L., d Stacy, E. W. Searching for objects in real-world scenes. Journal of Experimental Psychology. 1973, 97, 22-27.

ClaRK. H. H. The language-as-fixed-effect fallacy: A critique of language statistics in psychological research. Journal of Verbal Learning and Verbal Behavior, 1973, 12, 335-359.

Clark. H. H. Discussion of Wike and Church's comments. Journal of Verbal Learning and Verbal Behavior, 1976, 15, 257-261.

Ekman, P. Cross-cultural studies in facial expression. In $\mathbf{P}$. Ekman (Ed.), Darwin and facial expression. New York: Academic Press, 1973.

Homa, D., Haver, B., \& Schwartz, T. Perceptability of schematic face stimuli: Evidence for a perceptual Gestalt. Memory \& Cognition, 1976, 4, 176-185.

Reicher, G. Perceptual recognition as a function of meaningfulness of stimulus material. Journal of Experimental Psychology, 1969, 81, 275-280.

Trickett, W. H., Welch, B. L., \& James, G. S. Further critical values for the two-means problem. Biometrika, 1956, 43, 203-205.

Weisstein, N., \& Harris, C. S. Visual detection of line segments: An object superiority effect. Science, 1974, 186, 752-755

YIN, R. K. Looking at upside-down faces. Journal of Experimental Psychology, 1969, 81, 141-145.

YIN, R. K. Face recognition by brain injured patients: A dissociable ability? Neuropsychologia, 1970, 8, 395-402.

(Received for publication July 18, 1978.) 